\title{
HIGHLIGHT
}

\section{Paradigms of adult stem cell therapy for type 1 diabetes in mice}

Alexander V Kofman, Neil D Theise and Mehboob A Hussain

Liver and Stem Cell Research Laboratory, Beth Israel Medical Center, New York, NY and Albert Einstein Medical College, Bronx, NY, USA

(Correspondence should be addressed to Mehboob Hussain, Visiting Scientist, Liver and Stem Cell Research Laboratory, Division of Digestive Diseases, Beth Israel Medical Center, New York, NY, USA; Email: mehboobhussain@yahoo.com)

Hardly a day passes without news of stem cells. Stem cell therapy implies the replacement of diseased or lost cells from the progeny of stem cells that differentiate into the required phenotypes. Stem cells have the capacity to divide asymmetrically, that is, yielding one identical 'daughter' stem cell and a second daughter cell that proceeds towards terminal differentiation. Thus, stem cells are theroretically endlessly self-renewing and from thence derives their hoped-for uses. There are two large groups of stem cells: embryonic stem cells (stem cells derived from early embryonic stages) and adult stem cells (stem cells found in the postnatal organism).

Reconstitution of bone marrow by hematopoietic stem cell infusion is an established practice in the treatment of certain cancers and of certain disorders of hematopoiesis. Recently, bone marrow and other hematopoietic organs (liver and spleen) have been shown to harbor cells that possess the capacity to enter extramedullary tissues and to differentiate into functional parenchymal cells of the respective tissue, such as the liver, intestinal, skin and bronchial epithelia $(1,2)$, skeletal $(3,4)$, heart $(5,6)$ muscle and cells of the central nervous system $(7,8)$. These observations are not limited to experimental rodent models but are also made in biopsy material of human recipients of bone marrow transplantation $(9,10)$.

Several recent reports have uncovered the possibility of using adult stem cells from hematopoietic organs for the replacement of pancreatic endocrine cells and the treatment of type 1 diabetes mellitus (11-15). We summarize these findings as they represent different, albeit similar, paradigms of cellular regeneration in adult diabetic organisms.

In a first report, Ianus et al. (11) demonstrated that in mice, cells from transplanted bone marrow engraft into the pancreatic islets. A genetic mouse model allowed identification of transplanted bone marrowderived cells by virtue of a fluorescent protein that is expected to be expressed in cells if they transcribe insulin. Fluorescent cells were found in pancreatic islets of recipient mice approximately $1-2$ months after bone marrow transplantation. Importantly, fluorescent cells were also positive for the $\mathrm{Y}$ chromosome that came from the male donor in the female bone marrow recipient (Fig. 1A). These donor-derived engrafted cells express insulin and other genetic markers typical of pancreatic beta cells.

When tested in isolated culture conditions, the bone marrow-derived cells secrete insulin in response to a physiological glucose stimulus, and show intracellular calcium fluctuations reminiscent of pancreatic beta cells. However, 1-2 months after bone marrow transplantation, only about $1-3 \%$ of the islet cells originate from the transplanted bone marrow. This rate of engraftment does not account for the normal estimated turnover of 30 days of the endocrine cells in an islet (16). Additional studies demonstrated that cell fusion is not a likely explanation for the observed phenomenon of differentiation of bone marrow-derived cells into pancreatic endocrine cells.

In a second report by Hess et al. (12), streptozotocindiabetic mice were lethally irradiated and transplanted with bone marrow from donors that ubiquitously express green fluorescent protein (GFP). Whereas control mice remained hyperglycemic and showed increased mortality, bone marrow-transplanted mice were normoglycemic, had normal insulin production after 17 days and had an increased survival rate at day 42 (75$85 \%$ vs $0-50 \%)$. Increases in serum insulin correlated with reduction in blood glucose. Furthermore, the investigators showed that bone marrow cells that express c-kit - one of the 'stem cell markers' - as a surface marker could, when transplanted, lead to reduction in glycemia, whereas c-kit-negative cells had no effect. Comparison of engraftment into liver and pancreas revealed that bone marrow-derived cells engrafted preferentially into the streptozotocin-damaged pancreas.

In the pancreas, bone marrow-derived cells were found in two regions: the ducts and the islets. In the islets, most bone marrow-derived cells did not contain insulin, although a small number of bone marrowderived cells also contained insulin protein. These insulin-positive cells were found only in streptozotocin-diabetic bone marrow recipients. However, the insulin-positive cells did not coexpress the beta-cellspecific transcription factor PDX-1. The authors speculate that the insulin-positive cells may have been incorporating insulin from their surrounding environment rather than producing insulin themselves. Because of the small number of donor-derived, insulin-positive cells in the face of normoglycemia in bone 
A<smiles>C1CCCCCCC1</smiles>

"Transdifferentiation"

Pancreatic

endocrine islet cell

Bone marrow

derived stem cell

B

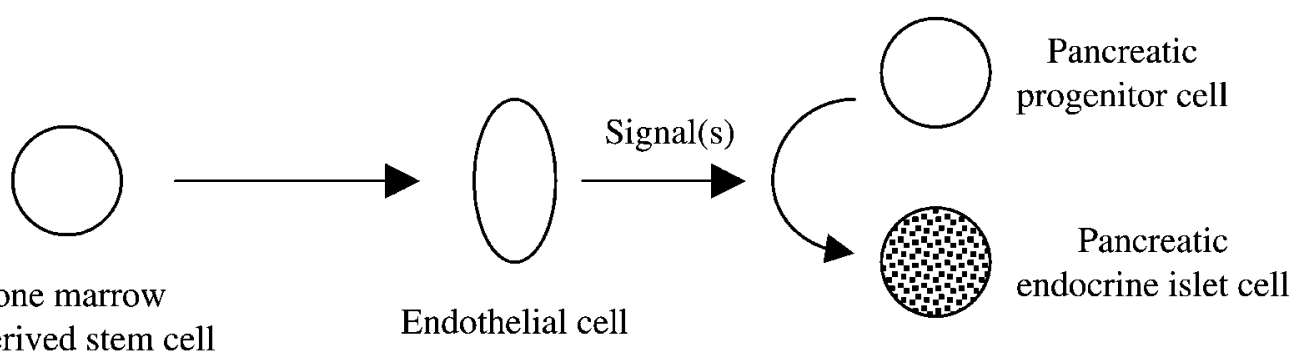
derived stem cell

(angioblast)

C<smiles>C1CCCCCCC1</smiles>
chimerism

Bone marrow derived cells
Autoimmune destruction stopped
Maintained normoglycemia

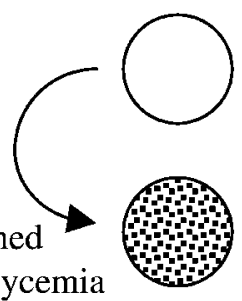

Pancreatic progenitor cell

Pancreatic endocrine islet cell

D

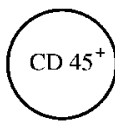
chimerism mesenchymal cell

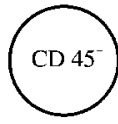$$
\text { chim }
$$

chimerism

"Transdifferentiation"
Autoimmune destruction stopped

Maintained

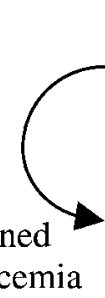
normoglycemia

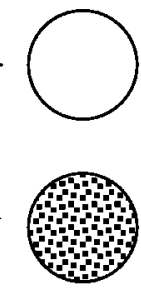

Pancreatic progenitor cell

Pancreatic endocrine islet cell
Autoimmune destruction stopped

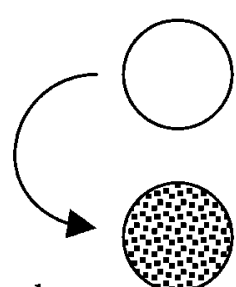

Maintained

normoglycemi

Figure 1 Schematic of proposed mechanisms underlying pancreatic islet cell regeneration after transplantation of cells derived from hematopoietic organs. (A) Direct differentiation (transdifferentiation) of bone marrow-derived stem cells into pancreatic endocrine cells (11). (B) Bone marrow contributes to generation of vascular endothelium. The newly formed endothelial cells stimulate differentiation of locally present host pancreatic progenitor cells (12). (C) Chimerism achieved by bone marrow transplantation in NOD mice induces immunologic tolerance towards beta cells. When euglycemia is maintained, local host pancreatic progenitor cells differentiate into endocrine cells (13). (D) Mesenchymal splenic cell transplantation plus treatment with complete Freund's adjuvant induces immunologic tolerance towards beta cells. Transplanted $\mathrm{CD} 45^{+}$splenic cells induce immune tolerance. When euglycemia is maintained, local progenitor cells differentiate into endocrine cells. Transplanted $\mathrm{CD} 45^{-}$splenic cells induce immune tolerance. When euglycemia is maintained, not only do local progenitor cells differentiate into endocrine cells, but CD45 ${ }^{-}$splenic cells also differentiate into pancreatic endocrine phenotypes $(14,15)$. 
marrow recipients, the authors further postulated that bone marrow-derived cells stimulate proliferation of host progenitor cells in the pancreas, leading to the increase in insulin-producing cell mass. Indeed, proliferation, as assessed by bromodeoxyuridine (BrdU) labeling, showed increased proliferation in bone marrow-recipient mice and virtually none in control animals.

In a subsequent step, the investigators determined that the bone marrow-derived cells had differentiated into endothelial cells (as determined by positive staining for PECAM-1, an endothelial protein) in the host pancreas. Taking these observations together, the authors propose that bone marrow cells home into damaged pancreatic islets, where they differentiate into endothelial cells. These cells in turn stimulate proliferation of locally present progenitor cells that then differentiate into pancreatic endocrine cells (Fig. 1B). This mechanism of endothelium-stimulated endocrine differentiation has previously been described in the developing pancreas (17).

Two additional groups (13-15) demonstrate the feasibility of cell therapy from hematopoietic organs in the nonobese diabetic (NOD) mouse, an autoimmune model of type 1 diabetes. The first group of investigators (13) used bone marrow transplantation to induce chimerism in NOD mice (Fig. 1C). In prediabetic NOD mice, microrchimerism prevented the development of autoimmune diabetes, presumably by mechanisms involving donor immunoregulatory cells that prevent the host cells from becoming autoreactive against beta cells. In NOD mice that have already developed diabetes (diabetic NOD mice), induction of microchimerism by sublethal or lethal irradiation followed bone marrow transplantation. In addition, these mice were maintained euglycemic by allotransplantation of islets under one kidney capsule or with exogenous insulin therapy. When, after 14 days, the kidney was removed, or the insulin therapy was stopped, the bone marrow transplant recipients remained euglycemic (cured).

BrdU analysis of pancreatic tissue of host animals revealed increased proliferative activity and regeneration of pancreatic beta cells. Furthermore, the islets that were transplanted under the kidney capsule and subsequently removed showed evidence of beta cell proliferation while the kidney was still in the animal. The authors speculate that the combination of bone marrow transplantation to induce immunologic silence and maintenance of euglycemia allowed beta cells or progenitor cells to proliferate as an adaptive response. The authors further consider 'transdifferentiation' of bone marrow cells into pancreatic endocrine cells as an additional possible mechanism to explain their observations. However, the methodology used in their studies does not allow consideration of this possibility.

Finally, Ryu et al. (14) and Kodama et al. (15) reported that the injection of mesenchymal splenocytes (mesenchymal cells from the spleen) and complete
Freund's adjuvant (CFA) into diabetic NOD mice eliminated autoimmunity and permanently restored normoglycemia (14). Furthermore, in these animals, the reversal of diabetes was accompanied by the reappearance of insulin-secreting islets in the pancreas. Recently, these authors have addressed the question of whether these newly formed islets are derived from transdifferentiation of donor nonlymphoid cells or from cells already present in the diabetic host animals (15) (Fig. 1D). Female diabetic NOD mice were treated with CFA and received transplants of either live or irradiated male mouse splenocytes. Blood sugar was controlled temporarily for 40 days by implanting syngeneic islets under the capsule of one of the kidneys. Six out of nine of the NOD mice that received live splenocytes remained normoglycemic after removal of the kidney with the islet transplants. In contrast, none of the animals that received irradiates splenocytes were normoglycemic after removal of the kidney with the islet transplants.

When normoglycemia was maintained for a longer period (120 days) before removal of the kidney carrying the islets, $11 / 12$ animals receiving live splenocytes and $11 / 13$ animals receiving irradiated splenocytes remained normoglycemic. Transplantation of both live and irradiated splenocytes led to long-term reversal of immune-mediated diabetes. There was no functional difference in the maintenance of normoglycemia between recipients of live versus irradiated splenocytes. However, the animals that had received irradiated splenocytes showed pronounced peri-insulitis, that is, lymphoid cells around but not in the newly formed islets, whereas no cellular infiltration was detectable in the recipients of live splenocytes. This coincided with the presence of donor-derived circulating blood cells $(4-12 \%)$ only in the peripheral blood of recipients of live splenocytes. Furthermore, with fluorescent in situ hybridization (FISH) for Y chromosome, male donor cells in long-term normoglycemic mice were found in abundance within islets, whereas the exocrine portions of the pancreas had few, if any, donor-derived cells. Male donor cells were also found in the epithelium of female NOD mouse pancreatic ducts. Ducts purely of host origin were not associated with adjacent islets containing male cells. No evidence of engraftment or transdifferentiation of donor cells was found in recipient brain, liver or kidney. By way of scanning focal lengths of donor-derived nuclei in beta cells, the authors further conclude that the donor-derived cells are not a result of fusion events. Very rare detection of cells with XXY and XXXY chromosomal complement in female recipient mice suggested the presence of few fusion events between donor and recipient cells.

In additional experiments, prediabetic female NOD mice received four times over 2 weeks transplantations of either $\mathrm{CD} 45^{+}, \mathrm{CD} 45^{-}$or unfractionated splenic mesenchymal cells expressing GFP. All subgroups remained normoglycemic. However, recipients of $\mathrm{CD} 45^{-}$or unfractionated GFP splenocytes revealed 
islets positive for the GFP marker. Prediabetic NOD females treated with $\mathrm{CD} 45^{+}$GFP splenic mesenchymal cells revealed no GFP in their islets. The latter animals had peri-insulitis. Thus, it appears that while $\mathrm{CD} 45^{+}$ splenic mesenchymal cells can influence disease activity, they do not include cells that participate directly in islet regeneration. Furthermore, these studies indicate that islet regeneration is taking place in prediabetic NOD mice, with participation of donor GFP mice and also host progenitor cells.

According to these results, it appears that $\mathrm{CD} 45^{-}$ splenic mesenchymal cells provide the double whammy of reversal of autoimmune disease as well as the cellular substrate for islet regeneration. However, as the results with the $\mathrm{CD} 45^{+}$cells demonstrate, keeping the insulitis at bay allows the yet to be identified endogenous (host) precursor cells to give rise to islet structures. It should be noted that fractionation of the splenic mesenchymal cells according to CD45 expression does not clearly identify which subpopulation of cells in the respective fraction is actually making the experiment work.

In humans, immunologic intervention to stop beta cell destruction early on during the development of type 1 diabetes mellitus allows for recovery of the pancreatic endocrine function $(18,19)$. This may perhaps be attributable to an increase in beta cell mass by recruitment of progenitor stem cells that can differentiate without succumbing to immune mediated destruction.
Several groups have recently identified pancreas resident progenitor cells that may give rise to endocrine islet cells. Human and rodent pancreatic duct cells (20-22), islet-derived progenitor cells $(22,23)$ and exocrine tissue (24) contain cells that can differentiate to some degree towards a pancreatic endocrine phenotype. Whether these cells were originally derived from a hematopoietic organ and are waiting in situ to differentiate on demand remains to be determined. However, it is likely that multiple pathways for regeneration exist, including resident, in-tissue, progenitors as well as extra tissue, circulating progenitors, usually marrow derived (25).

Some investigators indicate that cell fusion events are a possible mechanism, at least in part, for the phenomenon of transdifferentiation of bone marrow-derived cells into parenchymal cells of extramedullary organs $(26,27)$ (Fig. 2B). A few contend that cell fusion may be the only mechanism underlying apparent transdifferentiation $(28,29)$. The studies involving pancreatic endocrine cell regeneration, as outlined here, largely rule out cell fusion events as a mechanism of transdifferentiation $(11,15)$. There remains the possibility of cell fusion, the sharing of certain genetic and epigenetic information between fused cells, which then go apart with a newly phenotype adapted by the pluripotent cell (fusion followed by a reduction division) (Fig. 2C). However, for now, the majority of research papers do not support cell fusion as the main mechanism
A Stem cell

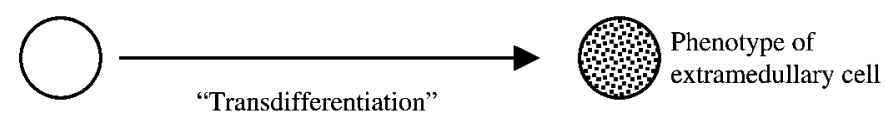

B Stem cell

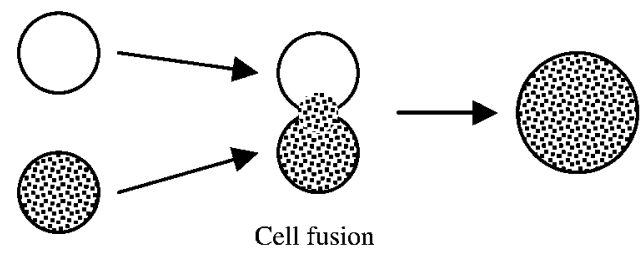

Result of fusion: tetraploid cell with certain phenotypic markers of differentiated cell

Differentiated cell

C Stem cell

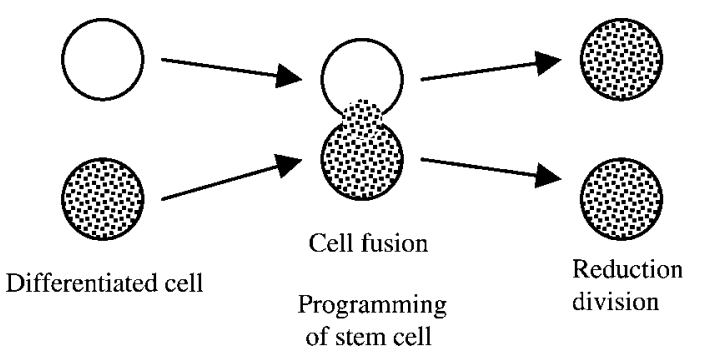

Two cells with differentiated phenotype
Figure 2 Schematic of proposed mechanism of adaptation of extramedullary phenotypes by cells derived from hematopoietic organs. (A) 'Stem cell' 'transdifferentitiates' into new functional extramedullary phenotype. (B) 'Stem cell' fuses with local differentiated cell. The tetraploid product of the fusion event has apparent phenotype of the differentiated cell. (C) 'Stem cell' and differentiated cell fuse. Information is exchanged between the cells, resulting in differentiation of the stem cell. Fusion is followed by a reduction division that separates the fused entities into two differentiated cells. 
underlying the adaptation of pancreatic phenotypes by circulating, largely bone marrow-derived cells (Fig. 2A).

\section{Acknowledgements}

M A H is a visiting scientist at the Beth Israel Medical Center, New York. N D T is supported by the Singer Hellman Foundation and the National Institutes of Health (NIH). M A H is supported by the American Diabetes Association, the Juvenile Diabetes Research Foundation and the NIH.

\section{References}

1 Krause DS, Theise ND, Collector MI, Henegariu O, Hwang S, Gardner $\mathrm{R}$ et al. Multi-organ, multi-lineage engraftment by a single bone marrow-derived stem cell. Cell $2001 \quad \mathbf{1 0 5}$ 369-377.

2 Petersen BE, Bowen WC, Patrene KD, Mars WM, Sullivan AK, Murase $\mathrm{N}$ et al. Bone marrow as a potential source of hepatic oval cells. Science $1999 \mathbf{2 8 4} 1168-1170$.

3 Ferrari G, Cusella-De Angelis G, Coletta M, Paolucci E Stornaiuolo A, Cossu G et al. Muscle regeneration by bone marrow-derived myogenic progenitors. Science $1998 \mathbf{2 7 0}$ $1528-1530$.

4 Gussoni E, Soneoka Y, Strickland CD, Buzney EA, Khan MK, Flint AF et al. Dystrophin expression in the mdx mouse restored by stem cell transplantation. Nature 1999401 360-394.

5 Orlic D, Kajstura J, Chimenti S, Jakoniuk I, Anderson SM, Li B et al. Bone marrow cells regenerate infracted myocardium. Nature $2001410701-705$

6 Jackson KA, Majka SM, Wang H, Pocius J, Hartley CJ, Majesky MW et al. Regeneration of ischemic cardiac muscle and vascular endothelium by adult stem cells. Journal of Clinica Investigation $2001 \mathbf{1 0 7} 1395-1402$.

7 Brazelton TRFM, Rossi GI, Kesher GI \& Blau HM. From marrow to brain: expression of neuronal phenotypes in adult mice. Science $20002901775-1779$

8 Mezey EKJ, Chandross G, Harta RA, Maki RA \& McKercher SR. Turning blood into brain: cells bearing neuronal antigens generated in vivo from bone marrow. Science 2000290 1779-1782.

9 Theise ND, Nimmakayalu M, Gardner R, Illei PB, Morgan G, Teperman L et al. Liver from bone marrow in humans. Hepatology $20003211-16$.

10 Körbling M, Katz RL, Khanna A, Ruifrok AC, Rondon G, Albitar M et al. Hepatocytes and epithelial cells of donor origin in recipients of peripheral blood stem cells. New England Journal of Medicine $2002346738-746$.

11 Ianus A, Holz GG, Theise ND \& Hussain MA. In vivo derivation of glucose-competent pancreatic endocrine cells from bone marrow without evidence of cell fusion. Journal of Clinical Investigation $2003111843-850$.

12 Hess D, Li L, Martin M, Sakano S, Hill D, Strutt B, Thyssen S, Gray DA \& Bhatia M. Bone marrow-derived stem cells initiate pancreatic regeneration. Nature Biotechnology 200321 $763-770$
13 Zorina TD, Subbotin VM, Bertera S, Alexander AM, Haluszczak C, Gambrell B, Bottino B, Styche AJ \& Trucco M. Recovery of endogenous $\beta$ cell function in the NOD model of autoimmune diabetes. Stem Cells 200321 377-388.

14 Ryu S, Kodama S, Ryu K, Schoenfeld DA \& Faustman DL. Reversal of established autoimmune diabetes by restoration of endogenous beta cell function. Journal of Clinical Investigation $2001 \mathbf{1 0 8}$ $63-72$.

15 Kodama S, Kühtreiber W, Fujimura S, Dala EA \& Faustman DL. Islet regeneration during the reversal of autoimmune diabetes in NOD mice. Science $2003 \mathbf{3 0 2} 1223-1226$.

16 Bonner-Weir S. Beta cell turnover: its assessment and implications. Diabetes 200150 (Supp l) S20-S24.

17 Lammert E, Cleaver O \& Melton D. Induction of pancreatic differentiation by signals from blood vessels. Science $2001 \mathbf{2 9 4}$ $564-567$.

18 Herold KC, Hagopian W, Auger JA, Poumian-Ruiz E, Taylor L, Donaldson D, Gitelman SE, Harlan DM, Xu D, Zivin RA \& Bluestone JA. Anti-CD3 monoclonal antibody in new-onset type 1 diabetes mellitus. New England Journal of Medicine $2002 \mathbf{3 4 6}$ $1692-1698$.

19 Glandt M, Hagopian W \& Herold KC. Treatment of type 1 diabetes with anti-CD3 monoclonal antibody. Review of Endocrine and Metabolic Disorders 20034 361-368.

20 Bonner-Weir S, Taneja M, Weir GC, Tatarkiewicz K. Song KH, Sharma A et al. In vitro cultivation of human islets from expanded ductal tissue. PNAS 200097 7999-8004.

21 Ramiya VK, Maraist M, Arfors KE, Schatz KA, Peck AB \& Cornelius JG. Reversal of insulin-dependent diabetes using islets generated in vitro from pancreatic stem cells. Nature Medicin $20006278-282$

22 Teitelman G. Induction of beta-cell neogenesis by islet injury. Diabetes Metabolism Reviews 199612 91-102.

23 Zulewski H, Abraham EJ, Gerlach MJ, Daniel PB, Moritz W, Muller B et al. Multipotential nestin-positive stem cells isolated from adult pancreatic islets differentiate ex vivo into pancreatic endocrine, exocrine, and hepatic phenotypes. Diabetes 200150 $521-533$.

24 Lipsett MD, Finegood D, Theise ND \& Wilmut I. Beta cell neogenesis during prolonged hyperglycemia in rats. Diabetes $2002 \mathbf{5 1}$ $1834-1841$.

25 Theise ND \& Wilmut I. Cell plasticity: flexible arrangement. Nature $2003 \mathbf{4 2 5} 21$

26 Terada D et al. Bone marrow cells adopt the phenotype of other cells by spontaneous cell fusion. Nature $2002 \mathbf{4 1 6} 542-545$

27 Alvarez-Dolado M, Pardal R, Garcia-Verdugo JM, Fike JR, Lee HO, Pfeffer K, Lois C, Morrison SJ \& Alvarez-Buylla A. Fusion of bonemarrow-derived cells with Purkinje neurons, cardiomyocytes and hepatocytes. Nature $2003 \mathbf{4 2 5} 968-973$.

28 Vassilopoulos G, Wang PR \& Russell DW. Transplanted bone marrow regenerates liver by cell fusion. Nature $2003 \mathbf{4 2 2}$ 901-904.

29 Wang X, Willenbring H, Akkari Y, Torimaru Y, Foster M, Al-Dhalimy M, Lagasse E, Finegold M, Olson S \& Grompe M. Cell fusion is the principal source of bone-marrow-derived hepatocytes. Nature $2003 \mathbf{4 2 2} 897-901$.

Received 15 January 2004

Accepted 16 January 2004 